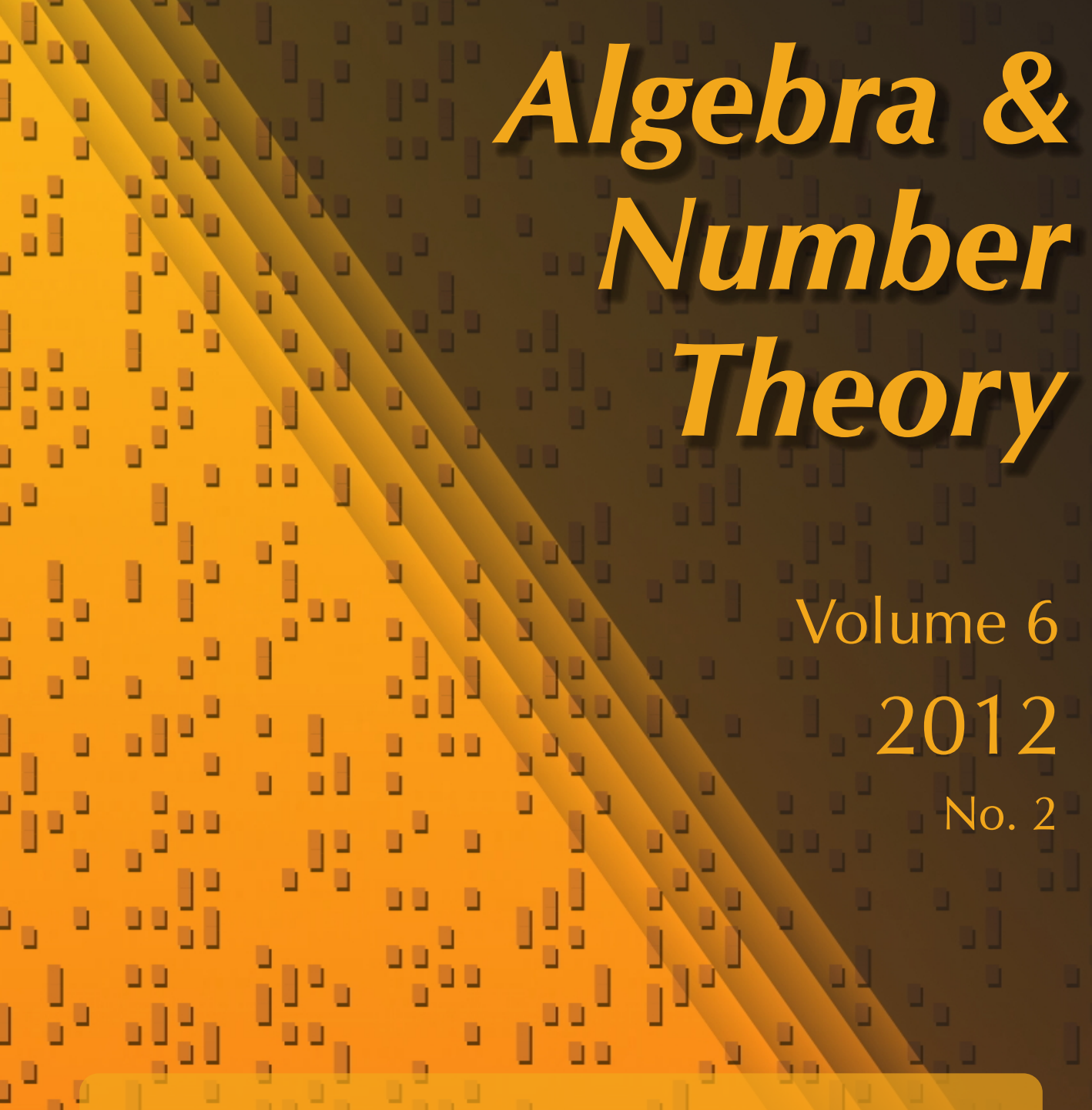

An upper bound on the Abbes-Saito filtration for finite flat group schemes $\downarrow \quad$ and applications

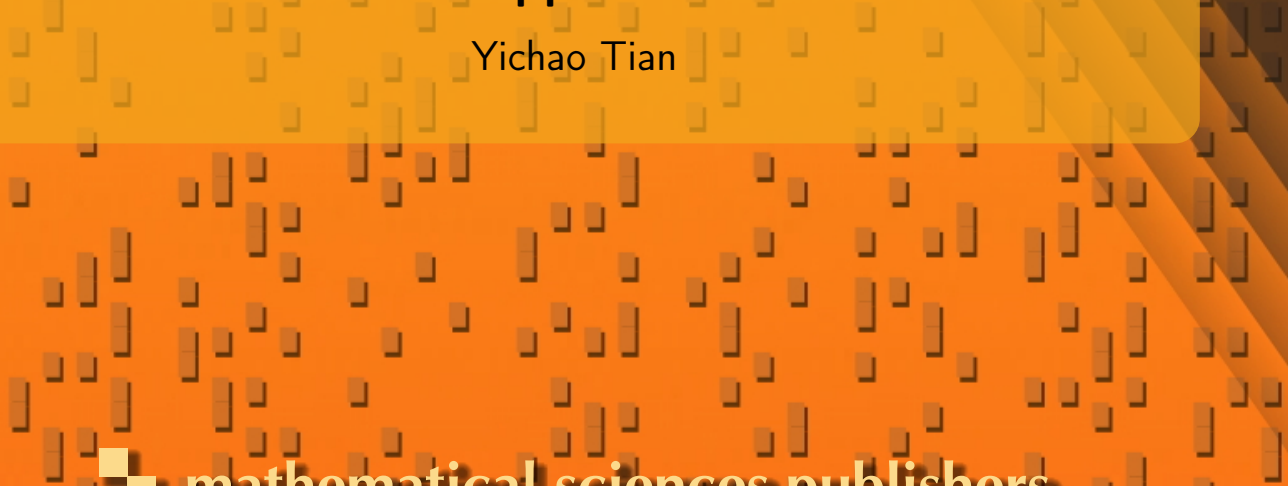

」

」」」」Jlume 6 


\title{
An upper bound on the Abbes-Saito filtration for finite flat group schemes and applications
}

\author{
Yichao Tian
}

Let $O_{K}$ be a complete discrete valuation ring of residue characteristic $p>0$, and $G$ be a finite flat group scheme over $0_{K}$ of order a power of $p$. We prove in this paper that the Abbes-Saito filtration of $G$ is bounded by a linear function of the degree of $G$. Assume $\mathbb{O}_{K}$ has generic characteristic 0 and the residue field of $\mathscr{O}_{K}$ is perfect. Fargues constructed the higher level canonical subgroups for a "near from being ordinary" Barsotti-Tate group $\mathscr{G}$ over $\mathfrak{O}_{K}$. As an application of our bound, we prove that the canonical subgroup of $\varphi$ of level $n \geq 2$ constructed by Fargues appears in the Abbes-Saito filtration of the $p^{n}$-torsion subgroup of $\mathscr{G}$.

Let $O_{K}$ be a complete discrete valuation ring with residue field $k$ of characteristic $p>0$ and fraction field $K$. We denote by $v_{\pi}$ the valuation on $K$ normalized by $v_{\pi}\left(K^{\times}\right)=\mathbb{Z}$. Let $G$ be a finite and flat group scheme over $\mathscr{O}_{K}$ of order a power of $p$ such that $G \otimes K$ is étale. We denote by $\left(G^{a}, a \in \mathbb{Q}_{\geq 0}\right)$ the Abbes-Saito filtration of $G$. This is a decreasing and separated filtration of $G$ by finite and flat closed subgroup schemes. We refer the readers to [Abbes and Saito 2002; 2003; Abbes and Mokrane 2004] for a full discussion, and to Section 1 for a brief review of this filtration. Let $\omega_{G}$ be the module of invariant differentials of $G$. The generic étaleness of $G$ implies that $\omega_{G}$ is a torsion $O_{K}$-module of finite type. Thus, there exist nonzero elements $a_{1}, \ldots, a_{d} \in \mathbb{O}_{K}$ such that

$$
\omega_{G} \simeq \bigoplus_{i=1}^{d} \mathcal{O}_{K} /\left(a_{i}\right) .
$$

We put $\operatorname{deg}(G)=\sum_{i=1}^{d} v_{\pi}\left(a_{i}\right)$, and call it the degree of $G$. The aim of this note is to prove the following:

Theorem 1. Let $G$ be a finite and flat group scheme over $\mathscr{O}_{K}$ of order a power of $p$ such that $G \otimes K$ is étale. Then we have $G^{a}=0$ for $a>p /(p-1) \operatorname{deg}(G)$.

This research was supported by a grant DMS-0635607 from the National Science Foundation. MSC2000: primary 14L15; secondary 14G22, 11S15.

Keywords: finite flat group schemes, ramification filtration, canonical subgroups. 
Our bound is optimal when $G$ is killed by $p$. Let $E_{\delta}=\operatorname{Spec}\left(O_{K}[X] /\left(X^{p}-\delta X\right)\right)$ be the group scheme of Tate-Oort over $0_{K}$. We have $\operatorname{deg}\left(E_{\delta}\right)=v_{\pi}(\delta)$, and an easy computation by Newton polygons gives [Fargues 2009, Lemme 5]:

$$
E_{\delta}^{a}= \begin{cases}E_{\delta} & \text { if } 0 \leq a \leq p /(p-1) \operatorname{deg}\left(E_{\delta}\right) \\ 0 & \text { if } a>p /(p-1) \operatorname{deg}\left(E_{\delta}\right)\end{cases}
$$

However, our bound may be improved when $G$ is not killed by $p$ or $G$ contains many identical copies of a closed subgroup. In [2006, Theorem 7], Hattori proves that if $K$ has characteristic 0 and $G$ is killed by $p^{n}$, then the Abbes-Saito filtration of $G$ is bounded by that of the multiplicative group $\mu_{p^{n}}$, i.e., we have $G^{a}=0$ if $a>e n+e /(p-1)$ where $e$ is the absolute ramification index of $K$. Compared with Hattori's result, our bound has the advantage that it works in both characteristic 0 and characteristic $p$, and that it is $\operatorname{good}$ if $\operatorname{deg}(G)$ is small.

The basic idea used to prove Theorem 1 is approximation of general power series over $\mathrm{O}_{K}$ by linear functions. First, we choose a "good" presentation of the algebra of $G$ such that the defining equations of $G$ involve only terms of total degree $m(p-1)+1$ with $m \in \mathbb{Z}_{\geq 0}$; see Proposition 1.6. The existence of such a presentation is a consequence of the classical theory on $p$-typical curves of formal groups. With this good presentation, we can prove in Lemma 1.9 that the neutral connected component of the $a$-tubular neighborhood of $G$ is isomorphic to a closed rigid ball for $a>p /(p-1) \operatorname{deg}(G)$, and the only zero of the defining equations of $G$ in the neutral component is the unit section.

The motivation of our theorem comes from the theory of canonical subgroups. We assume that $K$ has characteristic 0 , and the residue field $k$ is perfect of characteristic $p \geq 3$. Let $G$ be a Barsotti-Tate group of dimension $d \geq 1$ over $\mathscr{O}_{K}$. Abbes and Mokrane [2004] were the first to construct the canonical subgroup of level 1 of $G$ in the case where $G$ comes from an abelian scheme over $\mathcal{O}_{K}$. Then, Tian [2010] generalized their result to the Barsotti-Tate case. More specifically, it was shown that if a Barsotti-Tate group $G$ over $\mathscr{O}_{K}$ is "near from being ordinary", a condition expressed explicitly as a bound on the Hodge height of $G$ (see Section 2.1), then a certain piece of the Abbes-Saito filtration of $G[p]$ lifts the kernel of Frobenius of the special fiber of $G$ [Tian 2010, Theorem 1.4]. Later on, Fargues [2009] gave another construction of the canonical subgroup of level 1 using Hodge-Tate maps, and his approach also allowed us to construct by induction the canonical subgroups of level $n \geq 2$, i.e., the canonical lifts of the kernel of the $n$-th iteration of the Frobenius. He proved that the canonical subgroup of higher level appears in the Harder-Narasimhan filtration of $G\left[p^{n}\right]$, which was introduced by him in [Fargues 2007]. It is conjectured that the canonical subgroup of higher level also appears in the Abbes-Saito filtration of $G\left[p^{n}\right]$. In this paper, we prove this conjecture as a corollary, Theorem 2.5, of Theorem 1. Fargues's result on the degree of the 
quotient of $G\left[p^{n}\right]$ by its canonical subgroup of level $n$ (see Theorem 2.4(i)) will play an essential role in our proof.

Notation. In this paper, $O_{K}$ will denote a complete discrete valuation ring with residue field $k$ of characteristic $p>0$ and fraction field $K$. Let $\pi$ be a uniformizer of $\mathrm{O}_{K}$, and $v_{\pi}$ be the valuation on $K$ normalized by $v_{\pi}(\pi)=1$. Let $\bar{K}$ be an algebraic closure of $K, K^{\text {sep }}$ be the separable closure of $K$ contained in $\bar{K}$, and $\varphi_{K}$ be the Galois group $\operatorname{Gal}\left(K^{\text {sep }} / K\right)$. We also denote by $v_{\pi}$ the unique extension of the valuation to $\bar{K}$.

\section{Proof of Theorem 1}

First, we recall the definition of the filtration of Abbes-Saito for finite flat group schemes according to [Abbes and Mokrane 2004; Abbes and Saito 2003].

1.1. We denote the Jacobson radical of a semilocal ring $R$ by $\mathfrak{m}_{R}$. An algebra $R$ over $O_{K}$ is called formally of finite type if $R$ is semilocal, complete with respect to the $\mathfrak{m}_{R}$-adic topology, Noetherian, and $R / \mathfrak{m}_{R}$ is finite over $k$. We say an $\mathfrak{O}_{K^{-}}$ algebra $R$ formally of finite type is formally smooth if each of the factors of $R$ is formally smooth over $\mathrm{O}_{K}$.

Let FEA $_{\mathscr{O}_{K}}$ be the category of finite, flat, and generically étale $\mathscr{O}_{K}$-algebras, and Set $\mathscr{G}_{K}$ be the category of finite sets endowed with a discrete action of the Galois group $\varphi_{K}$. We have the fiber functor

$$
\mathscr{F}: \text { FEA }_{\bigcirc_{K}} \rightarrow \operatorname{Set}_{\varphi_{K}},
$$

which associates to an object $A$ of $\mathbf{F E} \mathbf{A}_{0_{K}}$ the set $\operatorname{Spec}(A)(\bar{K})$ equipped with the natural action of $\mathscr{G}_{K}$. We define a filtration on the functor $\mathscr{F}$ as follows. For each object $A$ in $\mathbf{F E A}_{\mathscr{O}_{K}}$, we choose a presentation

$$
0 \rightarrow I \rightarrow \mathscr{A} \rightarrow A \rightarrow 0
$$

where $\mathscr{A}$ is an $\mathbb{O}_{K}$-algebra formally of finite type and formally smooth. For any $a=m / n \in \mathbb{Q}_{>0}$ with $m$ prime to $n$, we define $\mathscr{A}^{a}$ to be the $\pi$-adic completion of the subring $\mathscr{A}\left[I^{n} / \pi^{m}\right] \subset \mathscr{A} \otimes_{\mathfrak{O}_{K}} K$ generated over $\mathscr{A}$ by all the $f / \pi^{m}$ with $f \in I^{n}$. The $\mathscr{O}_{K}$-algebra $\mathscr{A}^{a}$ is topologically of finite type, and the tensor product $\mathscr{A}^{a} \otimes_{\mathbb{O}_{K}} K$ is an affinoid algebra over $K$ [Abbes and Saito 2003, Lemma 1.4]. We put $X^{a}=\operatorname{Sp}\left(\mathscr{A}^{a} \otimes_{\mathcal{O}_{K}} K\right)$, which is a smooth affinoid variety over $K$ [Abbes and Saito 2003, Lemma 1.7]. We call it the $a$-th tubular neighborhood of $\operatorname{Spec}(A)$ with respect to the presentation (1). The $\varphi_{K}$-set of the geometric connected components of $X^{a}$, denoted by $\pi_{0}\left(X^{a}(A)_{\bar{K}}\right)$, depends only on the $\mathcal{O}_{K}$-algebra $A$ and the rational number $a$, but not on the choice of the presentation [Abbes and Saito 
2003, Lemma 1.9.2]. For rational numbers $b>a>0$, we have natural inclusions of affinoid varieties $\operatorname{Sp}\left(A \otimes_{0_{K}} K\right) \hookrightarrow X^{b} \hookrightarrow X^{a}$, which induce natural morphisms $\operatorname{Spec}(A)(\bar{K}) \rightarrow \pi_{0}\left(X^{b}(A)_{\bar{K}}\right) \rightarrow \pi_{0}\left(X^{a}(A)_{\bar{K}}\right)$. For a morphism $A \rightarrow B$ in FEA $_{\mathscr{O}_{K}}$, we can choose presentations of $A$ and $B$ so that we have a functorial map $\pi_{0}\left(X^{a}(B)_{\bar{K}}\right) \rightarrow \pi_{0}\left(X^{a}(A)_{\bar{K}}\right)$. Hence we get, for any $a \in \mathbb{Q}_{>0}$, a (contravariant) functor

$$
\mathscr{F}^{a}: \operatorname{FEA}_{\bigcirc_{K}} \rightarrow \operatorname{Set}_{\varphi_{K}}
$$

given by $A \mapsto \pi_{0}\left(X^{a}(A)_{\bar{K}}\right)$. We have natural morphisms of functors $\phi_{a}: \mathscr{F}_{F} \rightarrow \mathscr{F}^{a}$ and $\phi_{a, b}: \mathscr{F}^{b} \rightarrow \mathscr{F}^{a}$ for rational numbers $b>a>0$ with $\phi_{a}=\phi_{b, a} \circ \phi_{b}$. For any $A$ in $\mathbf{F E} \mathbf{A}_{\bigcirc_{K}}$, we have

$$
\mathscr{F}(A) \stackrel{\sim}{\rightarrow} \lim _{a \in \mathbb{Q}_{>0}} \mathscr{F}^{a}(A)
$$

[Abbes and Saito 2002, 6.4]; if $A$ is a complete intersection over $O_{K}$, the map $\mathscr{F}(A) \rightarrow \mathscr{F}^{a}(A)$ is surjective for any $a$ [Abbes and Saito 2002, 6.2].

1.2. Let $G=\operatorname{Spec}(A)$ be a finite and flat group scheme over $\mathscr{O}_{K}$ such that $G \otimes K$ is étale over $K$, and $a \in \mathbb{Q}_{>0}$. The group structure of $G$ induces a group structure on $\mathscr{F}^{a}(A)$, and the natural map $G(\bar{K})=\mathscr{F}(A) \rightarrow \mathscr{F}^{a}(A)$ is a homomorphism of groups. Hence, the kernel $G^{a}(\bar{K})$ of $G(\bar{K}) \rightarrow \mathscr{F}^{a}(A)$ is a $\mathscr{G}_{K}$-invariant subgroup of $G(\bar{K})$, and it defines a closed subgroup scheme $G_{K}^{a}$ of the generic fiber $G \otimes K$. The scheme theoretic closure of $G_{K}^{a}$ in $G$, denoted by $G^{a}$, is a closed subgroup of $G$ finite and flat over $\mathcal{O}_{K}$. Putting $G^{0}=G$, we get a decreasing and separated filtration $\left(G^{a}, a \in \mathbb{Q}_{\geq 0}\right)$ of $G$ by finite and flat closed subgroup schemes. We call it the Abbes-Saito filtration of $G$. For any real number $a \geq 0$, we put $G^{a+}=\bigcup_{b \in \mathbb{Q}_{>a}} G^{a}$. Assume $G$ is connected, i.e., the ring $A$ is local. Let

$$
0 \rightarrow I \rightarrow \mathrm{O}_{K} \llbracket X_{1}, \ldots, X_{d} \rrbracket \rightarrow A \rightarrow 0
$$

be a presentation of $A$ by the ring of formal power series such that the unit section of $G$ corresponds to the point $\left(X_{1}, \ldots, X_{d}\right)=(0, \ldots, 0)$. Since $A$ is a relative complete intersection over $\mathbb{O}_{K}, I$ is generated by $d$ elements $f_{1}, \ldots, f_{d}$. For $a \in \mathbb{Q}_{>0}$, the $\bar{K}$-valued points of the $a$-th tubular neighborhood of $G$ are given by

$$
X^{a}(\bar{K})=\left\{\left(x_{1}, \ldots, x_{d}\right) \in \mathfrak{m}_{\bar{K}}^{d} \mid v_{\pi}\left(f_{i}\left(x_{1}, \ldots, x_{d}\right)\right) \geq a \text { for } 1 \leq i \leq d\right\},
$$

where $\mathfrak{m}_{\bar{K}}$ is the maximal ideal of $\mathcal{O}_{\bar{K}}$. The subset $G(\bar{K}) \subset X^{a}(\bar{K})$ corresponds to the zeros of the $f_{i}$ 's. Let $X_{0}^{a}$ be the connected component of $X^{a}$ containing 0 . Then the subgroup $G^{a}(\bar{K})$ is the intersection of $X_{0}^{a}(\bar{K})$ with $G(\bar{K})$.

The basic properties of Abbes-Saito filtration that we need are summarized as follows. 
Proposition 1.3 [Abbes and Mokrane 2004, 2.3.2, 2.3.5]. Let $G$ and $H$ be finite and flat group schemes, generically étale over $O_{K}$, and $f: G \rightarrow H$ be a homomorphism of group schemes.

(i) The closed subgroup $G^{0+}$ is the connected component of $G$, and we have $\left(G^{0+}\right)^{a}=G^{a}$ for any $a \in \mathbb{Q}_{>0}$.

(ii) Given a $\in \mathbb{Q}_{>0}, f$ induces a canonical homomorphism $f^{a}: G^{a} \rightarrow H^{a}$. If $f$ is flat and surjective, then $f^{a}(\bar{K}): G^{a}(\bar{K}) \rightarrow H^{a}(\bar{K})$ is surjective.

Now we return to the proof of Theorem 1 .

Lemma 1.4. Let $R$ be a $\mathbb{Z}_{p}$-algebra, $\mathscr{X}$ be a formal group of dimension $d$ over $R$ such that $\mathrm{Lie}(\mathscr{X})$ is a free $R$-module of rank $d$. Then

(i) the ring $\mathbb{Z}_{p}$ acts naturally on $\mathscr{X}$, and its image in $\operatorname{End}_{R}(\mathscr{X})$ lies in the center of $\operatorname{End}_{R}(\mathscr{X})$;

(ii) there exist parameters $\left(X_{1}, \ldots, X_{d}\right)$ of $\mathscr{X}$ such that

$$
[\zeta]\left(X_{1}, \ldots, X_{d}\right)=\left(\zeta X_{1}, \ldots, \zeta X_{d}\right)
$$

for any $(p-1)$-st root of unity $\zeta \in \mathbb{Z}_{p}$.

Proof. This is actually a classical result on formal groups. In the terminology of [Hazewinkel 1978], the formal group $\mathscr{X}$ comes from the base change of $\mathscr{X}^{\text {univ }}$ defined by the $d$-dimensional universal $p$-typical formal group law (denoted by $F_{V}(X, Y)$ in [Hazewinkel 1978, 15.2.8]) over

$$
\mathbb{Z}_{p}[V]=\mathbb{Z}_{p}\left[V_{i}(j, k) ; i \in \mathbb{Z}_{\geq 0}, j, k=1, \ldots, d\right],
$$

where the $V_{i}(j, k)$ are free variables. So we are reduced to proving the lemma for $\mathscr{Q}^{\text {univ }}$. If $X$ and $Y$ stand for the column vectors $\left(X_{1}, \ldots, X_{d}\right)$ and $\left(Y_{1}, \ldots, Y_{d}\right)$ respectively, the formal group law on $\mathscr{X}^{\text {univ }}$ is determined by

$$
F_{V}(X, Y)=f_{V}^{-1}\left(f_{V}(X)+f_{V}(Y)\right), \quad \text { with } f_{V}(X)=\sum_{i=0}^{\infty} a_{i}(V) X^{p^{i}},
$$

where the $a_{i}(V)$ are certain $d \times d$ matrices with coefficients in $\mathbb{Q}_{p}[V]$ with $a_{1}(V)$ invertible, $X^{p^{i}}$ stands for $\left(X_{1}^{p^{i}}, \ldots, X_{d}^{p^{i}}\right)$, and $f_{V}^{-1}$ is the unique $d$-tuple of power series in $\left(X_{1}, \ldots, X_{d}\right)$ with coefficients in $\mathbb{Q}_{p}[V]$ such that $f_{V}^{-1} \circ f_{V}=1$; see [Hazewinkel 1978, 10.4]. We note that $F_{V}(X, Y)$ is a $d$-tuple of power series with coefficient in $\mathbb{Z}_{p}[V]$, although $f_{V}(X)$ has coefficients in $\mathbb{Q}_{p}[V]$ [Hazewinkel 1978, 10.2(i)]. Via approximation by integers, we see easily that the operation of multiplication by an element $\xi \in \mathbb{Z}_{p}$ given by $[\xi](X)=f_{V}^{-1}\left(\xi f_{V}(X)\right)$ is well defined. This proves (i). Statement (ii) is an immediate consequence of the fact that $f_{V}(X)$ contains only $p$-powers of $X$. 
Remark 1.5. The referee gives the following alternative proof of this lemma via the Cartier theory of formal groups. Let $\mathscr{X}$ be the formal group over $R$ as in the lemma. We denote by $\mathscr{X}(R \llbracket T \rrbracket)$ the group of $R \llbracket T \rrbracket$-valued points of $\mathscr{L}$ whose reduction modulo $T$ is the neutral element $0 \in \mathscr{L}(R)$. A formal group law over $\mathscr{X}$ is a datum $\left(\mathscr{X} ; \gamma_{1}, \ldots, \gamma_{d}\right)$, where $\gamma_{1}, \ldots, \gamma_{d} \in \mathscr{X}(R \llbracket T \rrbracket)$ are such that their image in $\mathscr{X}\left(R[T] / T^{2}\right)$ forms a basis for $\operatorname{Lie}(\mathscr{X})$. In particular, $\left(\gamma_{i}\right)_{1 \leq i \leq d}$ establish an isomorphism $\mathscr{X} \simeq \operatorname{Spf}\left(R \llbracket X_{1}, \ldots, X_{d} \rrbracket\right)$ of formal schemes over $R$. Recall that $\mathscr{X}(R \llbracket T \rrbracket)$ is the Cartier module associated with $\mathscr{X}$ over the big Cartier ring (denoted by $\operatorname{Cart}(R)$ in [Chai 2004, 2.3]). Since $R$ is a $\mathbb{Z}_{p}$-algebra, the Cartier theory [Chai 2004, 4.3, 4.4] implies that there exists a $p$-typical formal group law $\left(\mathscr{X} ; \gamma_{1}, \ldots, \gamma_{d}\right)$ over $\mathscr{X}$, i.e., we have $\epsilon_{p} \cdot \gamma_{i}=0$, where

$$
\epsilon_{p}=\prod_{\substack{\ell \text { prime } \\(\ell, p)=1}}\left(1-\frac{1}{\ell} V_{\ell} F_{\ell}\right)
$$

is Cartier's idempotent in $\operatorname{Cart}(R)$; see [Chai 2004, 4.1]. Let $\Delta: \mathbb{Z}_{p}=W\left(\mathbf{F}_{p}\right) \rightarrow$ $W\left(\mathbb{Z}_{p}\right)$ be the Cartier homomorphism given by $\left(x_{0}, x_{1}, \ldots\right) \mapsto\left(\left[x_{0}\right],\left[x_{1}\right], \ldots\right)$, where $x_{n} \in \mathbf{F}_{p}$ and $\left[x_{n}\right]$ denotes its Teichmüller lift. Then we get a natural map $u: \mathbb{Z}_{p} \stackrel{\Delta}{\rightarrow} W\left(\mathbb{Z}_{p}\right) \rightarrow W(R)$. For a $(p-1)$-st root of unity $\zeta \in \mathbb{Z}_{p}$, we have $u(\zeta)=$ $[\zeta] \in W(R)$. Note that for any $a \in R$ and $1 \leq i \leq d$, the $p$-typical curve $[a] \cdot \gamma_{i}$ is the image of $\gamma_{i}$ under the map $\mathscr{L}(R \llbracket T \rrbracket) \rightarrow \mathscr{L}(R \llbracket T \rrbracket)$ induced by $T \mapsto a T$. Applying this fact to $u(\zeta) \cdot \gamma_{i}=[\zeta] \cdot \gamma_{i}$, one obtains the lemma immediately.

Proposition 1.6. Let $G=\operatorname{Spec}(A)$ be a connected finite and flat group scheme over $\mathrm{O}_{K}$ of order a power of $p$. Then there exists a presentation of $A$ of type (2) such that the defining equations $f_{i}$ for $1 \leq i \leq d$ have the form

$$
f_{i}\left(X_{1}, \ldots, X_{d}\right)=\sum_{|n| \geq 1}^{\infty} a_{i, \underline{n}} X^{\underline{n}} \quad \text { with } a_{i, \underline{n}}=0 \text { if }(p-1) \nmid(|\underline{n}|-1),
$$

where $\underline{n}=\left(n_{1}, \ldots, n_{d}\right) \in\left(\mathbb{Z}_{\geq 0}\right)^{d}$ are multiindexes, $|\underline{n}|=\sum_{j=1}^{d} n_{j}$, and $X^{\underline{n}}$ is short for $\prod_{j=1}^{\bar{d}} X_{j}^{n_{j}}$.

Proof. By a theorem of Raynaud [Berthelot et al. 1982, 3.1.1], there is a projective abelian variety $V$ over $\mathrm{O}_{K}$, and an embedding of group schemes $j: G \hookrightarrow V$. Let $V^{\prime}$ be the quotient of $V$ by $G$. Let $\mathscr{X}$, $\mathscr{Y}$ be, respectively, the formal completions of $V$ and $V^{\prime}$ along their unit sections. They are formal groups over $0_{K}$. Since $G$ is connected, it is identified with the kernel of the natural isogeny $\phi: \mathscr{X} \rightarrow \mathscr{Y}$. Let $\left(X_{1}, \ldots, X_{d}\right)$ (respectively $\left.\left(Y_{1}, \ldots, Y_{d}\right)\right)$ be parameters of $\mathscr{X}$ (respectively $\mathscr{y}$ ) satisfying the preceding lemma. The isogeny $\phi$ is thus given by

$$
\left(X_{1}, \ldots, X_{d}\right) \mapsto\left(f_{1}\left(X_{1}, \ldots, X_{d}\right), \ldots, f_{d}\left(X_{1}, \ldots, X_{d}\right)\right),
$$


where $f_{i}=\sum_{|\underline{n}| \geq 1} a_{i, \underline{n}} X^{\underline{n}} \in \mathcal{O}_{K} \llbracket X_{1}, \ldots, X_{d} \rrbracket$. Since for any $(p-1)$-th root of unity $\zeta \in \mathbb{Z}_{p}$ we have $f_{i}\left(\zeta X_{1}, \ldots, \zeta X_{d}\right)=\zeta f_{i}\left(X_{1}, \ldots, X_{d}\right)$, it's easy to see that $a_{i, \underline{n}}=0$ if $(p-1) \nmid(|\underline{n}|-1)$.

Remark 1.7. As pointed out by the referee, we can avoid using Raynaud's deep theorem to realize $G$ as the kernel of an isogeny of formal groups over $\mathscr{O}_{K}$. In fact, by the biduality formula $G \simeq\left(G^{D}\right)^{D}$, where $G^{D}$ denotes the Cartier dual of $G$, we have a canonical closed embedding $u: G \hookrightarrow U=\operatorname{Res}_{G^{D} / S}\left(\mathbf{G}_{m}\right)$ of group schemes over $S=\operatorname{Spec}\left(O_{K}\right)$. Here, "Res $G^{D} / S$ " means Weil's restriction of scalars, so $U$ is an affine smooth group scheme over $S$. Since the quotient of an affine scheme by a finite flat group scheme is always representable by a scheme [Raynaud 1967], we can consider the quotient $U^{\prime}=U / G$ and the formal groups $\mathscr{X}$, $\mathscr{Y}$ associated with $U$ and $U^{\prime}$, so that $G$ is the kernel of the natural isogeny $\phi: \mathscr{X} \rightarrow \mathscr{Y}$.

1.8. Proof of Theorem 1. Let $H=G^{0+}$ be the connected component of $G$. By 1.3(i), we have $G^{a}=H^{a}$ for $a \in \mathbb{Q}_{>0}$. The exact sequence of finite flat group schemes $0 \rightarrow H \rightarrow G \rightarrow G / H \rightarrow 0$ induces a long exact sequence of finite $\mathrm{O}_{K^{-}}$ modules

$$
0 \rightarrow H^{-1}\left(\ell_{G / H}\right) \rightarrow H^{-1}\left(\ell_{G}\right) \rightarrow H^{-1}\left(\ell_{H}\right) \rightarrow \omega_{G / H} \rightarrow \omega_{G} \rightarrow \omega_{H} \rightarrow 0,
$$

where $\ell_{G}$ means the co-Lie complex of $G$ [Berthelot et al. 1982, 3.2.9]. Since the generic fiber of $G / H$ is étale, it's easy to see that Thus, it follows that $0 \rightarrow$ $\omega_{G / H} \rightarrow \omega_{G} \rightarrow \omega_{H} \rightarrow 0$ is exact. Since $G / H$ is étale, we have $\omega_{G / H}=0$ and hence $\operatorname{deg}(G)=\operatorname{deg}(H)$. Up to replacing $G$ by $H$, we may assume that $G=\operatorname{Spec}(A)$ is connected.

We choose a presentation of $A$ as in Proposition 1.6 so that we have an isomorphism of $\mathrm{O}_{K}$-algebras

$$
A \simeq \mathfrak{O}_{K} \llbracket X_{1}, \ldots, X_{d} \rrbracket /\left(f_{1}, \ldots, f_{d}\right)
$$

where

$$
f_{i}\left(X_{1}, \ldots, X_{d}\right)=\sum_{j=1}^{d} a_{i, j} X_{j}+\sum_{|\underline{n}| \geq p} a_{i, \underline{n}} X^{\underline{n}} .
$$

As $A$ is finite as an $O_{K}$-module, we have

$$
\Omega_{A / \mathbb{O}_{K}}^{1}=\widehat{\Omega}_{A / \mathbb{O}_{K}}^{1} \simeq\left(\bigoplus_{i=1}^{d} A d X_{i}\right) /\left(d f_{1}, \ldots, d f_{d}\right) .
$$

Since $\omega_{G} \simeq e^{*}\left(\Omega_{A / \mathbb{O}_{K}}^{1}\right)$, where $e$ is the unit section of $G$, we get

$$
\omega_{G} \simeq\left(\bigoplus_{i=1}^{d} \mathcal{O}_{K} d X_{i}\right) /\left(\sum_{1 \leq j \leq d} a_{i, j} d X_{j}\right)_{1 \leq i \leq d} .
$$


In particular, if $U$ denotes the matrix $\left(a_{i, j}\right)_{1 \leq i, j \leq d}$, then $\operatorname{deg}(G)=v_{\pi}(\operatorname{det}(U))$.

For any rational number $\lambda$, we denote by $\mathbf{D}^{d}\left(0,|\pi|^{\lambda}\right)\left(\right.$ respectively $\left.\mathbb{D}^{d}\left(0,|\pi|^{\lambda}\right)\right)$ the rigid analytic closed (respectively open) disk of dimension $d$ over $K$ consisting of points $\left(x_{1}, \ldots, x_{d}\right)$ with $v_{\pi}\left(x_{i}\right) \geq \lambda$ (respectively $\left.v_{\pi}\left(x_{i}\right)>\lambda\right)$ for $1 \leq i \leq d$; we put $\mathbf{D}^{d}(0,1)=\mathbf{D}^{d}\left(0,|\pi|^{0}\right)$ and $\mathbb{D}^{d}(0,1)=\mathbb{\mathbb { D }}^{d}\left(0,|\pi|^{0}\right)$. Let $a>p /(p-1) \operatorname{deg}(G)$ be a rational number, $X^{a}$ be the $a$-th tubular neighborhood of $G$ with respect to the chosen presentation. By (3), we have a cartesian diagram of rigid analytic spaces

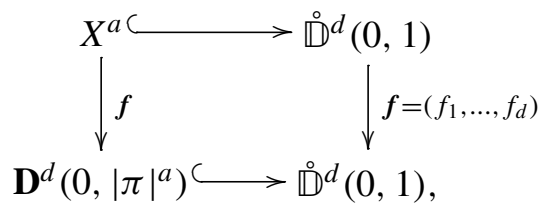

where $\boldsymbol{f}\left(y_{1}, \ldots, y_{d}\right)=\left(f_{1}\left(y_{1}, \ldots, y_{d}\right), \ldots, f_{d}\left(y_{1}, \ldots, y_{d}\right)\right)$ and horizontal arrows are inclusions. Let $X_{0}^{a}$ be the connected component of $X^{a}$ containing 0 . By the discussion below (3), we just need to prove that 0 is the only zero of the $f_{i}$ contained in $X_{0}^{a}$.

Let $V=\left(b_{i, j}\right)_{1 \leq i, j \leq d}$ be the unique $d \times d$ matrix with coefficients in $\mathbb{O}_{K}$ such that $U V=V U=\operatorname{det}(U) I_{d}$, where $I_{d}$ is the $d \times d$ identity matrix. If $\mathbf{A}_{K}^{d}$ denotes the $d$-dimensional rigid affine space over $K$, then $V$ defines an isomorphism of rigid spaces

$$
\boldsymbol{g}: \mathbf{A}_{K}^{d} \rightarrow \mathbf{A}_{K}^{d}, \quad\left(x_{1}, \ldots, x_{d}\right) \mapsto\left(\sum_{j=1}^{d} b_{1, j} x_{j}, \ldots, \sum_{j=1}^{d} b_{d, j} x_{j}\right) .
$$

It's clear that $\boldsymbol{g}\left(\mathbb{\mathbb { D }}^{d}(0,1)\right) \subset \mathbb{\mathbb { D }}^{d}(0,1)$, so that $\boldsymbol{f}$ is defined on $\boldsymbol{g}\left(\mathbb{\mathbb { D }}^{d}(0,1)\right)$. The composite morphism $\boldsymbol{f} \circ \boldsymbol{g}: \mathbb{\mathbb { D }}^{d}(0,1) \rightarrow \mathbb{\mathbb { D }}^{d}(0,1)$ is given by

$$
\left(x_{1}, \ldots, x_{d}\right) \mapsto\left(\operatorname{det}(U) x_{1}+R_{1}, \ldots, \operatorname{det}(U) x_{d}+R_{d}\right),
$$

where $R_{i}=\sum_{|\underline{n}| \geq p} a_{i, \underline{n}} \prod_{j=1}^{d}\left(\sum_{k=1}^{d} b_{j, k} x_{k}\right)^{n_{j}}$ involves only terms of order $\geq p$ for $1 \leq i \leq d$. For $1 \leq i \leq d$, we have basic estimations

$$
v_{\pi}\left(\operatorname{det}(U) x_{i}\right)=\operatorname{deg}(G)+v_{\pi}\left(x_{i}\right) \quad \text { and } \quad v_{\pi}\left(R_{i}\right) \geq p \min _{1 \leq j \leq d}\left\{v_{\pi}\left(x_{j}\right)\right\} .
$$

Lemma 1.9. For any rational number $a>p /(p-1) \operatorname{deg}(G)$, the map $\boldsymbol{g}$ induces an isomorphism of affinoid rigid spaces

$$
\boldsymbol{g}: \mathbf{D}^{d}\left(0,|\pi|^{a-\operatorname{deg}(G)}\right) \stackrel{\sim}{\longrightarrow} X_{0}^{a} .
$$

Assuming this lemma for a moment, we can complete the proof of Theorem 1 as follows. Consider the composite

$$
\boldsymbol{h}=\left.\boldsymbol{f} \circ \boldsymbol{g}\right|_{\mathbf{D}^{d}\left(0,|\pi|^{a-\operatorname{deg}(G)}\right)}: \mathbf{D}^{d}\left(0,|\pi|^{a-\operatorname{deg}(G)}\right) \stackrel{\sim}{\rightarrow} X_{0}^{a} \hookrightarrow X^{a} \stackrel{f}{\rightarrow} \mathbf{D}^{d}\left(0,|\pi|^{a}\right) .
$$


To complete the proof of Theorem 1, we just need to prove that $\boldsymbol{h}^{-1}(0)=\{0\}$. Let $\left(x_{1}, \ldots, x_{d}\right)$ be a point of $\mathbf{D}^{d}\left(0,|\pi|^{a-\operatorname{deg}(G)}\right)$, and $\left(z_{1}, \ldots, z_{d}\right)=\boldsymbol{h}\left(x_{1}, \ldots, x_{d}\right)$. We may assume $v_{\pi}\left(x_{1}\right)=\min _{1 \leq i \leq d}\left\{v_{\pi}\left(x_{i}\right)\right\}$. We have $v_{\pi}\left(x_{1}\right) \geq a-\operatorname{deg}(G)>$ $1 /(p-1) \operatorname{deg}(G)$ by the assumption on $a$. It follows thus from (6) that

$$
v_{\pi}\left(R_{1}\right) \geq p v_{\pi}\left(x_{1}\right)>\operatorname{deg}(G)+v_{\pi}\left(x_{1}\right)=v_{\pi}\left(\operatorname{det}(U) x_{1}\right) .
$$

Hence, we deduce from (5) that $v_{\pi}\left(z_{1}\right)=\operatorname{deg}(G)+v_{\pi}\left(x_{1}\right)$. In particular, $z_{1}=0$ if and only if $x_{1}=0$. Therefore, we have $\boldsymbol{h}^{-1}(0)=\{0\}$. This achieves the proof of Theorem 1.

Proof of Lemma 1.9. Let $\epsilon$ be any rational number with

$$
0<\epsilon<(p-1) / p a-\operatorname{deg}(G) .
$$

We will prove that

$$
\mathbf{D}^{d}\left(0,|\pi|^{a-\operatorname{deg}(G)}\right)=\mathbf{D}^{d}\left(0,|\pi|^{a-\operatorname{deg}(G)-\epsilon}\right) \cap \boldsymbol{g}^{-1}\left(X^{a}\right) .
$$

This will imply that $\mathbf{D}^{d}\left(0,|\pi|^{a-\operatorname{deg}(G)}\right)$ is a connected component of $\boldsymbol{g}^{-1}\left(X^{a}\right)$. Since $\boldsymbol{g}: \mathbf{A}_{K}^{d} \rightarrow \mathbf{A}_{K}^{d}$ is an isomorphism, the lemma will follow immediately.

We prove first the inclusion $\subset$. It suffices to show $\boldsymbol{g}\left(\mathbf{D}^{d}\left(0,|\pi|^{a-\operatorname{deg}(G)}\right)\right) \subset X^{a}$. Let $\left(x_{1}, \ldots, x_{d}\right)$ be a point of $\mathbf{D}^{d}\left(0,|\pi|^{a-\operatorname{deg}(G)}\right)$. By (4), we have to check that $\left(z_{1}, \ldots, z_{d}\right)=\boldsymbol{f}\left(\boldsymbol{g}\left(x_{1}, \ldots, x_{d}\right)\right)$ lies in $\mathbf{D}^{d}\left(0,|\pi|^{a}\right)$. We obtain from (6) that $v_{\pi}\left(\operatorname{det}(U) x_{i}\right)=\operatorname{deg}(G)+v_{\pi}\left(x_{i}\right) \geq a$ and $v_{\pi}\left(R_{i}\right) \geq p(a-\operatorname{deg}(G))$. As $a>$ $p /(p-1) \operatorname{deg}(G)$, we have $v_{\pi}\left(R_{i}\right)>a$. It follows from (5) that

$$
v_{\pi}\left(z_{i}\right) \geq \min \left\{v_{\pi}\left(\operatorname{det}(U) x_{i}\right), v_{\pi}\left(R_{i}\right)\right\} \geq a .
$$

This proves $\left(z_{1}, \ldots, z_{d}\right) \subset \mathbf{D}^{d}\left(0,|\pi|^{a}\right)$; hence $\boldsymbol{g}\left(\mathbf{D}^{d}\left(0,|\pi|^{a-\operatorname{deg}(G)}\right)\right) \subset X^{a}$.

To prove the inclusion $\supset$, we just need to verify that every point which is in $\mathbf{D}^{d}\left(0,|\pi|^{a-\operatorname{deg}(G)-\epsilon}\right)$ but outside $\mathbf{D}^{d}\left(0,|\pi|^{a-\operatorname{deg}(G)}\right)$ does not lie in $\boldsymbol{g}^{-1}\left(X^{a}\right)$. Let $\left(x_{1}, \ldots, x_{d}\right)$ be such a point. We may assume that

$a-\operatorname{deg}(G)-\epsilon \leq v_{\pi}\left(x_{1}\right)<a-\operatorname{deg}(G) \quad$ and $\quad v_{\pi}\left(x_{i}\right) \geq a-\operatorname{deg}(G)-\epsilon$ for $2 \leq i \leq d$.

Let

$$
\left(z_{1}, \ldots, z_{d}\right)=\left(\operatorname{det}(U) x_{1}+R_{d}, \ldots, \operatorname{det}(U) x_{d}+R_{d}\right)
$$

be the image of $\left(x_{1}, \ldots, x_{d}\right)$ under the composite $f \circ g$. According to (4), the proof will be completed if we can prove that $\left(z_{1}, \ldots, z_{d}\right)$ is not in $\mathbf{D}^{d}\left(0,|\pi|^{a}\right)$. From (6) and (7), we get $v_{\pi}\left(\operatorname{det}(U) x_{1}\right)=\operatorname{deg}(G)+v_{\pi}\left(x_{1}\right)<a$ and $v_{\pi}\left(R_{1}\right) \geq$ $p(a-\operatorname{deg}(G)-\epsilon)$. Thanks to the assumption on $\epsilon$, we have $p(a-\operatorname{deg}(G)-\epsilon)>a$, so $v_{\pi}\left(z_{1}\right)=v_{\pi}\left(\operatorname{det}(U) x_{1}\right)<a$. This shows that $\left(z_{1}, \ldots, z_{d}\right)$ is not in $\boldsymbol{g}^{-1}\left(X^{a}\right)$; hence the proof of the lemma is complete. 


\section{Applications to canonical subgroups}

In this section, we suppose the fraction field $K$ has characteristic 0 and the residue field $k$ is perfect of characteristic $p \geq 3$. Let $e$ be the absolute ramification index of $\mathbb{O}_{K}$. For any rational number $\epsilon>0$, we denote by $\mathbb{O}_{K, \epsilon}$ the quotient of $\mathscr{O}_{K}$ by the ideal consisting of elements with $p$-adic valuation greater or equal to $\epsilon$.

2.1. First we recall some results on the from [Abbes and Mokrane 2004; Tian 2010; Fargues 2009]. Let $v_{p}: \mathscr{O}_{K} / p \rightarrow[0,1]$ be the truncated $p$-adic valuation (with $v_{p}(0)=1$ ). Let $G$ be a truncated Barsotti-Tate group of level $n \geq 1$ nonétale over $\mathscr{O}_{K}$, and $G_{1}=G \otimes_{\mathscr{O}_{K}}\left(\mathscr{O}_{K} / p\right)$. The Lie algebra of $G_{1}$ denoted by $\operatorname{Lie}\left(G_{1}\right)$ is a finite free $\mathscr{O}_{K} / p$-module. The Verschiebung homomorphism $V_{G_{1}}: G_{1}^{(p)} \rightarrow G_{1}$ induces a semilinear endomorphism $\varphi_{G_{1}}$ of $\operatorname{Lie}\left(G_{1}\right)$. We choose a basis of $\operatorname{Lie}\left(G_{1}\right)$ over $0_{K} / p$, and let $U$ be the matrix of $\varphi$ under this basis. We define the Hodge height of $G$, denoted by $h(G)$, to be the truncated $p$-adic valuation of $\operatorname{det}(U)$. We note that the definition of $h(G)$ does not depend on the choice of $U$. The Hodge height of $G$ is an analog of the Hasse invariant in mixed characteristic, and we have $h(G)=0$ if and only if $G$ is ordinary.

Theorem 2.2 [Fargues 2009, théorème 4]. Let $G$ be a truncated Barsotti-Tate group of level 1 over $O_{K}$ of dimension $d \geq 1$ and height $h$. Assume $h(G)<1 / 2$ if $p \geq 5$ and $h(G)<1 / 3$ if $p=3$.

(i) For any rational number ep/( $p-1) h(G)<a \leq e p /(p-1)(1-h(G))$, the finite flat subgroup $G^{a}$ of $G$ given by the Abbes-Saito filtration has rank $p^{d}$.

(ii) Let $C$ be the subgroup $G^{e p /(p-1)(1-h(G))}$ of $G$. We have $\operatorname{deg}(G / C)=e h(G)$.

(iii) The subgroup $C \otimes \mathfrak{O}_{K, 1-h(G)}$ coincides with the kernel of the Frobenius homomorphism of $G \otimes \mathbb{O}_{K, 1-h(G)}$. Moreover, for any rational number $\epsilon$ with $h(G) /(p-1)<\epsilon \leq 1-h(G)$, if $H$ is a finite and flat closed subgroup of $G$ such that $H \otimes \mathbb{O}_{K, \epsilon}$ coincides with the kernel of Frobenius of $G \otimes \mathcal{O}_{K, \epsilon}$, then we have $H=C$.

The subgroup $C$ in this theorem, when it exists, is called the canonical subgroup (of level 1 ) of $G$.

Remark 2.3. The conventions here are slightly different from those in [Fargues 2009]. The Hodge height is called Hasse invariant there, while we choose to follow the terminologies in [Abbes and Mokrane 2004] and [Tian 2010]. Our index of Abbes-Saito filtration and the degree of $G$ are $e$ times those in [Fargues 2009].

Part (iii) of Theorem 2.2 is not explicitly stated in [Fargues 2009, théorème 4], but it's an easy consequence of Proposition 11 in that paper.

For the canonical subgroups of higher level, we have this: 
Theorem 2.4 [Fargues 2009, théorème 6]. Let $G$ be a truncated Barsotti-Tate group of level $n$ over $\mathcal{O}_{K}$ of dimension $d \geq 1$ and height $h$. Assume $h(G)<1 / 3^{n}$ if $p=3$ and $h(G)<1 /\left(2 p^{n-1}\right)$ if $p \geq 5$.

(i) There exists a unique closed subgroup of $G$ that is finite and flat over $\mathbb{O}_{K}$ and satisfies the following:

- $C_{n}(\bar{K})$ is free of rank $d$ over $\mathbb{Z} / p^{n} \mathbb{Z}$.

- For each integer $i$ with $1 \leq i \leq n$, let $C_{i}$ be the scheme theoretic closure of $C_{n}(\bar{K})\left[p^{i}\right]$ in $G$. Then the subgroup $C_{i} \otimes \mathcal{O}_{K, 1-p^{i-1} h(G)}$ coincides with the kernel of the $i$-th iterated Frobenius of $G \otimes \mathcal{O}_{K, 1-p^{i-1} h(G)}$.

(ii) We have $\operatorname{deg}\left(G / C_{n}\right)=e\left(p^{n}-1\right) /(p-1) h(G)$.

The subgroup $C_{n}$ in the theorem above is called the canonical subgroup of level $n$ of $G$. Fargues actually proves that $C_{n}$ is a certain piece of the Harder-Narasimhan filtration of $G$. The aim of this section is to show that $C_{n}$ appears also in the Abbes-Saito filtration.

Theorem 2.5. Let $G$ be a truncated Barsotti-Tate group of level $n$ over $\mathbb{O}_{K}$ satisfying the assumptions in Theorem 2.4, and $C_{n}$ be its canonical subgroup of level $n$. Then for any rational number a satisfying

$$
e p\left(p^{n}-1\right) /(p-1)^{2} h(G)<a \leq e p /(p-1)(1-h(G)),
$$

we have $G^{a}=C_{n}$.

Proof. We proceed by induction on $n$. If $n=1$, this is Theorem 2.2(i). We suppose $n \geq 2$ and the theorem is valid for truncated Barsotti-Tate groups of level $n-1$. For each integer $i$ with $1 \leq i \leq n$, let $G_{i}$ denote the scheme theoretic closure of $G(\bar{K})\left[p^{i}\right]$ in $G$, and $C_{i}$ the scheme theoretic closure of $C_{n}(\bar{K})\left[p^{i}\right]$ in $C_{n}$. By Theorem 2.4(i), it's clear that $C_{i}$ is the canonical subgroup of level $i$ of $G_{i}$. Let $a$ be a rational number with $\left(e p\left(p^{n}-1\right) /(p-1)^{2}\right) h(G)<a \leq(e p /(p-1))(1-h(G))$. By the induction hypothesis and the functoriality of Abbes-Saito filtration 1.3(ii), we have $C_{n-1}(\bar{K})=G_{n-1}^{a}(\bar{K}) \subset G^{a}(\bar{K})$, and the image of $G^{a}(\bar{K})$ in $G_{1}(\bar{K})$ is exactly $C_{1}(\bar{K})=G_{1}^{a}(\bar{K})$. Note that we have a commutative diagram

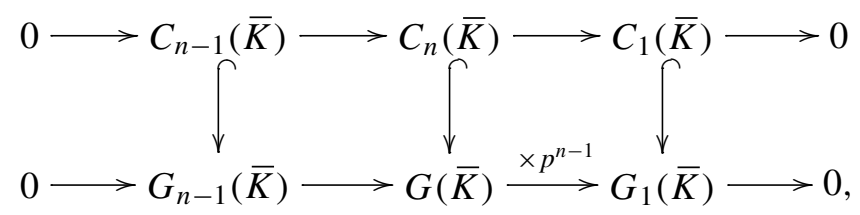

where the rows are exact sequences of groups and the vertical arrows are natural inclusions. So we have $C_{n}(\bar{K}) \subset G^{a}(\bar{K})$. On the other hand, Theorems 1 and 2.4(ii) 
imply that $\left(G / C_{n}\right)^{a}(\bar{K})=0$ since

$$
a>\frac{e p\left(p^{n}-1\right)}{(p-1)^{2}} h(G)=\frac{p}{p-1} \operatorname{deg}\left(G / C_{n}\right) .
$$

Therefore, we get $G^{a}(\bar{K}) \subset C_{n}(\bar{K})$ by Proposition 1.3(ii). This completes the proof.

\section{Acknowledgements}

I would like to thank Ahmed Abbes for his comments on an earlier version of this paper. I also express my deep gratitude to the anonymous referee for his careful reading and useful suggestions for clarifying some arguments.

\section{References}

[Abbes and Mokrane 2004] A. Abbes and A. Mokrane, "Sous-groupes canoniques et cycles évanescents $p$-adiques pour les variétés abéliennes", Publ. Math. Inst. Hautes Études Sci. 99 (2004), 117162. MR 2005f:14090 Zbl 1062.14057

[Abbes and Saito 2002] A. Abbes and T. Saito, "Ramification of local fields with imperfect residue fields", Amer. J. Math. 124:5 (2002), 879-920. MR 2003m:11196 Zbl 1084.11064

[Abbes and Saito 2003] A. Abbes and T. Saito, "Ramification of local fields with imperfect residue fields, II”, Doc. Math. Extra Vol. (2003), 5-72. MR 2005g:11231 Zbl 1127.11349

[Berthelot et al. 1982] P. Berthelot, L. Breen, and W. Messing, Théorie de Dieudonné cristalline, II, Lecture Notes in Mathematics 930, Springer, Berlin, 1982. MR 85k:14023 Zbl 0516.14015

[Chai 2004] C. L. Chai, "Notes on Cartier-Dieudonné theory", 2004, available at http://tinyurl.com/ 74 bcra7.

[Fargues 2007] L. Fargues, "La filtration de Harder-Narasimhan des schémas en groupes finis et plats", preprint, 2007. To appear in J. Reine Angew. Math.

[Fargues 2009] L. Fargues, "La filtration canonique des points de torsion des groupes $p$-divisibles", preprint, 2009, available at http://www-irma.u-strasbg.fr/ fargues/canoniqueHN.pdf.

[Hattori 2006] S. Hattori, "Ramification of a finite flat group scheme over a local field", J. Number Theory 118:2 (2006), 145-154. MR 2007b:14104 Zbl 1107.14036

[Hazewinkel 1978] M. Hazewinkel, Formal groups and applications, Pure and Applied Mathematics 78, Academic Press, New York, 1978. MR 82a:14020 Zbl 0454.14020

[Raynaud 1967] M. Raynaud, "Passage au quotient par une relation d'équivalence plate”, pp. 78-85 in Proc. Conf. Local Fields (Driebergen, 1966), edited by T. A. Springer, Springer, Berlin, 1967. MR 38 \#1104 Zbl 0165.24003

[Tian 2010] Y. Tian, "Canonical subgroups of Barsotti-Tate groups", Ann. of Math. (2) 172:2 (2010), 955-988. MR 2012a:14105 Zbl 1203.14026

Communicated by Brian Conrad

Received 2010-05-03 Revised 2011-05-02 Accepted 2011-05-30

yichaot@math.ac.cn

Mathematics Department, Fine Hall, Washington Road, Princeton, NJ 08544, United States

Current address: 


\section{Algebra \& Number Theory}

msp.berkeley.edu/ant

\section{EDITORS}

MANAGING EDITOR

Bjorn Poonen

Massachusetts Institute of Technology

Cambridge, USA

\author{
EDITORIAL BOARD CHAIR \\ David Eisenbud \\ University of California \\ Berkeley, USA
}

\section{BOARD OF EDITORS}

Georgia Benkart

Dave Benson

Richard E. Borcherds

John H. Coates

J-L. Colliot-Thélène

Brian D. Conrad

Hélène Esnault

Hubert Flenner

Edward Frenkel

Andrew Granville

Joseph Gubeladze

Ehud Hrushovski

Craig Huneke

Mikhail Kapranov

Yujiro Kawamata

János Kollár

Yuri Manin

Barry Mazur

Philippe Michel

Susan Montgomery
University of Wisconsin, Madison, USA

University of Aberdeen, Scotland

University of California, Berkeley, USA

University of Cambridge, UK

CNRS, Université Paris-Sud, France

University of Michigan, USA

Universität Duisburg-Essen, Germany

Ruhr-Universität, Germany

University of California, Berkeley, USA

Université de Montréal, Canada

San Francisco State University, USA

Hebrew University, Israel

University of Kansas, USA

Yale University, USA

University of Tokyo, Japan

Princeton University, USA

Northwestern University, USA

Harvard University, USA

École Polytechnique Fédérale de Lausanne

University of Southern California, USA
Shigefumi Mori

Raman Parimala

Jonathan Pila

Victor Reiner

Karl Rubin

Peter Sarnak

Joseph H. Silverman

Michael Singer

Ronald Solomon

Vasudevan Srinivas

J. Toby Stafford

Bernd Sturmfels

Richard Taylor

Ravi Vakil

Michel van den Bergh

Marie-France Vignéras

Kei-Ichi Watanabe

Andrei Zelevinsky

Efim Zelmanov
RIMS, Kyoto University, Japan

Emory University, USA

University of Oxford, UK

University of Minnesota, USA

University of California, Irvine, USA

Princeton University, USA

Brown University, USA

North Carolina State University, USA

Ohio State University, USA

Tata Inst. of Fund. Research, India

University of Michigan, USA

University of California, Berkeley, USA

Harvard University, USA

Stanford University, USA

Hasselt University, Belgium

Université Paris VII, France

Nihon University, Japan

Northeastern University, USA

University of California, San Diego, USA

\section{PRODUCTION}

contact@msp.org

Silvio Levy, Scientific Editor

See inside back cover or www.jant.org for submission instructions.

The subscription price for 2012 is US \$175/year for the electronic version, and \$275/year (+\$40 shipping outside the US) for print and electronic. Subscriptions, requests for back issues from the last three years and changes of subscribers address should be sent to Mathematical Sciences Publishers, Department of Mathematics, University of California, Berkeley, CA 94720-3840, USA.

Algebra \& Number Theory (ISSN 1937-0652) at Mathematical Sciences Publishers, Department of Mathematics, University of California, Berkeley, CA 94720-3840 is published continuously online. Periodical rate postage paid at Berkeley, CA 94704, and additional mailing offices.

ANT peer review and production are managed by EditFLOW ${ }^{\circledR}$ from Mathematical Sciences Publishers.

PUBLISHED BY

mathematical sciences publishers

http://msp.org/

A NON-PROFIT CORPORATION

Typeset in IAT $_{\mathrm{E}} \mathrm{X}$

Copyright (C2012 by Mathematical Sciences Publishers 


\section{Algebra \& Number Theory}

Volume $6 \quad$ No. $2 \quad 2012$

Arithmetic of singular Enriques surfaces

Klaus HuleK and MatThias SchÜtT

An upper bound on the Abbes-Saito filtration for finite flat group schemes and applications

YICHAO TIAN

On the smallest number of generators and the probability of generating an algebra

Rostyslav V. Kravchenko, Marcin Mazur and Bogdan V. Petrenko

Moving lemma for additive higher Chow groups

AMALENDU KRISHNA and JINHYUN PARK

CHRISTOPHER GOFF

Uniformly rigid spaces

CHRISTIAN KAPPEN

On a conjecture of Kontsevich and Soibelman

LÊ QUY THUONG 